JOURNAL OF THE

AMERICAN MATHEMATICAL SOCIETY

Volume 14, Number 1, Pages 109-124

S 0894-0347(00)00350-7

Article electronically published on September 20, 2000

\title{
SUPERSIMPLE THEORIES
}

\author{
STEVEN BUECHLER, ANAND PILLAY, AND FRANK WAGNER
}

\section{INTRODUCTION}

Suppose $M$ is a structure in the sense of model theory. It is natural to consider definable quotients $X / E$, where $X$ is a definable set in $M$ and $E$ a definable equivalence relation. An element $a / E$ of $X / E$ is called an imaginary element of $M$. Shelah introduced the formalisation $M^{e q}$, a many-sorted first order structure where such quotients and their elements can be treated on an equal footing with ordinary definable sets and elements. The passage to $M^{e q}$ was required in stability theory to understand "canonical bases" of types (the stability-theoretic generalisation of the smallest field of definition of a variety).

One can go further and consider sets $X / E$ where $E$ is now a "type-definable" equivalence relation, namely defined by a possibly infinite conjunction of first order formulas. We call an element $a / E$ of $X / E$ a "hyperimaginary", and $X / E$ a hyperdefinable set. To really see $X / E$ one should assume the structure $M$ to be sufficiently saturated. Hyperimaginaries naturally arise in nonstandard analysis, by taking $E(x, y)$ to be the relation " $|a-b|$ is infinitesimal". On the other hand if $M$ is stable, then it was pointed out in [14 that a type-definable equivalence relation is essentially a conjunction of definable equivalence relations, whereby a hyperimaginary is no more than a possibly infinite sequence (or projective limit) of imaginaries (we call this phenomenon elimination of hyperimaginaries).

In the past few years, the class of simple theories, a far-reaching generalisation of the class of stable theories, has been intensively studied. As vector spaces and algebraically closed fields are natural examples of stable theories (or rather structures), so vector spaces over finite fields equipped with bilinear forms, pseudofinite fields, and algebraically closed fields with a generic automorphism are examples of simple unstable theories. Simple theories were introduced by Shelah in his fundamental paper [16, and defined using his notion of "forking". In 7], major progress was made by showing that in simple theories, forking behaves very much like in stable theories, in particular provides a good notion of independence. (See also [8], [9].) Further advances were made in 12, where among other things the Independence Theorem for Lascar strong types was proved. This is a theorem concerning the compatibility of various free extensions, and was motivated by earlier work of

Received by the editors July 14, 1999 and, in revised form, June 20, 2000.

2000 Mathematics Subject Classification. Primary 03C45.

The first author was supported by an NSF grant. The second author was supported by an NSF grant and thanks MSRI for its hospitality. The third author was supported by DFG grant Wa 899/2-1 and thanks MSRI for its hospitality.

(C)2000 American Mathematical Society 
Hrushovski ([5]) in a certain finite-dimensional context. The interesting phenomenon was that, in place of the definable equivalence relations with finitely many classes (corresponding to strong types) which occur in stability theory, we seemed to be forced to consider type-definable equivalence relations with a small number of classes (corresponding to Lascar strong types). In a similar vein, in [4, in trying to develop the theory of canonical bases (something like fields of definition of varieties) in the simple theory context, one was forced to consider hyperimaginaries, rather than just imaginaries. (Related work is in [2].) However the mystery was that we did not know (and still do not know) any example of a simple theory in which hyperimaginaries are not eliminable. Supersimple theories (or structures) are simple structures in which the independence relation given by forking is finitely based. All the examples mentioned earlier are supersimple. The purpose of the current paper is to prove that in a supersimple theory (or structure) hyperimaginaries are eliminable. Equivalently, any equivalence relation defined by a possibly infinite conjunction of formulas is essentially an intersection of definable equivalence relations. A special case of our result, when $E$ has a small number of classes, is that (in a supersimple structure) any hyperdefinable compact group $G$ which acts definably as a group of automorphisms of some hyperdefinable set $X$ has to be profinite.

We now mention related results as well as the genesis of the current paper (which is a bit complicated). Kim [10] proved that in any countable theory with only countably many types, any type-definable equivalence relation (over a finite set) is a conjunction of definable equivalence relations. Hrushovski ([6]) showed that if one was willing to work in suitable non-first-order contexts (so-called Robinson structures), then there are supersimple theories which do not eliminate (even bounded) hyperimaginaries. Buechler [1] proved that in simple theories satisfying an additional technical property (lowness) any type-definable equivalence relation with few classes is a conjunction of definable finite equivalence relations (at least on the set of realisations of a complete type). This was also proved by Shami [15]. Buechler generalised his methods in [2], suggesting a proof that, for supersimple low theories, canonical bases (slightly different from those in the present paper) exist as sets of imaginaries. Hart and Pillay [3] shortened the proof as well as translating it into the context of [4. (However both 2] and [3] had gaps.) Using these ideas, as well as an analysability strategy developed a year earlier at the Fields Institute, Wagner suggested a proof for elimination of hyperimaginaries in the general supersimple context. This was subsequently simplified by Pillay and Wagner and is essentially the proof presented here.

The remainder of this paper assumes some acquaintance with model theory and its language. In section 2 we give definitions and background results on simple theories and hyperimaginaries, as well as some additional results. The proof of the main result (elimination of hyperimaginaries for supersimple theories) is given in section 5. The proof is quite technical and depends on the availability of a lot of machinery (such as analysability, orthogonality) in the hyperimaginary context. So for the sake of motivation and for the benefit of the reader, in section 3 we outline a rather elementary case of the argument, which in particular yields the main result in the supersimple low case. The reader familiar with the essentials of stability and simplicity theory could read section 3 first if he or she so desired. In section 4 we develop internality and analysability for hyperimaginaries, as well as introducing a rather useful analysability rank $R$. With this technology available, we then prove the main result in section 5 . 


\section{Preliminaries}

We start by recalling definitions and results regarding simple theories and hyperimaginaries. The basic material on simple theories is from [16], [7, 12] and also [1] for low theories. The material on hyperimaginaries and canonical bases is from 4] and [13. But there are also a few new observations.

$T$ denotes a complete theory in a language $L$, and $\bar{M}$ a $\bar{\kappa}$ - saturated model of $T$ for some suitable large $\bar{\kappa}$. "Small" means of cardinality strictly less than $\bar{\kappa}$. There is no harm in working in $\bar{M}^{e q}$ in which there are additional sorts $S_{E}$ whenever $E(x, y)$ is a formula of $L$ defining an equivalence relation on $\bar{M}^{n}$. Until we say otherwise $x, y$, etc. will denote possibly infinite but small tuples of variables, each variable ranging over a sort $S_{E}$. Similarly, $a, b$ denote small tuples of elements and $A, B$ small subsets of $\bar{M}$ (or $\bar{M}^{e q}$ ). A partial type in variables $x$ is a small consistent collection of formulas in some variables $x$ and various parameters. We write $\Sigma(x, a)$ for such a partial type if we want to specify the parameters as coming from $a$.

Definition 2.1. $\Sigma(x, b)$ divides over $A$ if there is an $A$-indiscernible sequence $\left(b_{i}: i<\omega\right)$ in $\operatorname{tp}(b / A)$ such that $\bigcup\left\{\Sigma\left(x, b_{i}\right): i<\omega\right\}$ is inconsistent (is not realised in $\bar{M})$.

Definition 2.2. $T$ is simple if whenever $a$ is a finite tuple and $B$ any set, then there is a subset $A$ of $B$ of cardinality at most $|T|$ such that $t p(a / B)$ does not divide over $A$.

Remark 2.3. (i) For simple theories, dividing is equivalent to a certain apriori weaker notion, forking. For simple theories we use these words interchangeably.

(ii) For $T$ simple, forking is a good independence relation, satisfying symmetry, transitivity, and existence of nonforking extensions, even for infinite tuples. For example symmetry means $t p(a / A b)$ does not fork over $A$ iff $t p(b / A a)$ does not fork over $A$. Existence of nonforking extensions means, for any $a, A$ and $A \subseteq B$ there is $a^{\prime}$ such that $t p\left(a^{\prime} / A\right)=t p(a / A)$ and $t p\left(a^{\prime} / B\right)$ does not fork over $A$. We also use the expression: $a$ is independent from $b$ over $A$ to mean $t p(a / A b)$ does not fork over $A$.

(iii) Note that $t p(a / B)$ does not fork over $A$ iff for each finite subtuple $a^{\prime}$ of $a$, $t p\left(a^{\prime} / B\right)$ does not fork over $A$.

(iv) Suppose $\left\{a_{i}: i \in I\right\}$ is a set of tuples. We call this set $A$-independent if for each $i \in I, a_{i}$ is independent from $\left\{a_{j}: j \in I, j \neq i\right\}$ over $A$.

Definition 2.4. (i) Let $\psi(x)$ be a formula with maybe additional parameters, $\phi(x, y)$ and $L$-formula, and $k<\omega . D(\psi(x), \phi(x, y), k)$ is defined to be $\geq 0$ if $\psi(x)$ has a solution, and $\geq n+1$ if there are $b_{i}$ for $i<\omega$ such that $\left\{\phi\left(x, b_{i}\right)\right.$ : $i<\omega\}$ is $k$-inconsistent (every $k$-sized subset is inconsistent) and for each $i$, $D\left(\psi(x) \wedge \phi\left(x, b_{i}\right), \phi, k\right) \geq n$.

(ii) For $\Sigma(x)$ a partial type closed under conjunctions, $D(\Sigma, \phi(x, y), k)$ is defined to be $\min \{D(\psi(x), \phi(x, y), k): \psi(x) \in \Sigma(x)\}$ if this min. exists (and $\infty$ otherwise).

Fact 2.5. (i) If $T$ is simple, then for any partial type $\Sigma(x)$ (in finitely many variables $x), \phi(x, y) \in L$ and $k<\omega, D(\Sigma, \phi, k)$ exists (namely has value $<\omega$ ). Moreover, if $p(x) \subset q(x)$ are complete types over $A \subset B$ respectively, then $q$ does not fork over $A$ (is a nonforking extension of $p$ ) iff for each $\phi(x, y)$ and $k$, $D(p, \phi, k)=D(q, \phi, k)$.

(ii) For fixed $\psi(x, z), \phi(x, y), k, n$, there is a set $\Gamma(z)$ of formulas such that $D(\psi(x, c), \phi, k) \geq n$ iff $\models \Gamma(c)$. 
(iii) It follows that for $T$ simple, and $p(x)$ a complete type (even in infinitely many variables) over $A$, there is a partial type $\Sigma\left(x_{i}\right)_{i \in I}$ over $A$ such that for any set $\left\{c_{i}: i \in I\right\}$ of tuples, this is an A-independent set of realisations of $p$ iff $=\Sigma\left(c_{i}\right)_{i}$.

(iv) (T simple.) Let $\Sigma(x)$ be a partial type over $B \supset A$ which does not fork over $A$. Then $\Sigma(x)$ extends to a complete type $p(x)$ over $B$ which does not fork over $A$.

Definition 2.6 ( $T$ simple). Let $p(x) \in S(A)$. By a Morley sequence in $p$ we mean an infinite $A$-independent $A$-indiscernible sequence $\left(c_{i}: i<\omega\right)$ of realisations of $p(x)$.

Fact 2.7 ( $T$ simple). Let $\Sigma(x, b)$ be a partial type, and $A$ a set. Then $\Sigma(x, b)$ does not fork over $A$ iff for any Morley sequence $\left(b_{i}: i<\omega\right)$ in $\operatorname{tp}(b / A), \bigcup\left\{\Sigma\left(x, b_{i}\right): i<\right.$ $\omega\}$ is consistent, iff for some Morley sequence $\left(b_{i}: i<\omega\right)$ in $\operatorname{tp}(b / A), \bigcup\left\{\Sigma\left(x, b_{i}\right)\right.$ : $i<\omega\}$ is consistent.

Definition 2.8. The formula $\phi(x, y)$ is said to be low, if there is some $k<\omega$ such that whenever $\left(b_{i}: i<\omega\right)$ is indiscernible and $\left\{\phi\left(x, b_{i}\right): i<\omega\right\}$ is inconsistent, then it is $k$-inconsistent. $T$ is low iff all formulas $\phi(x, y)$ are low. (Note the similarity to the non fcp.)

Definition 2.9 ( $T$ simple or, more generally, " $G$-compact"). Let $a, b$ be possibly infinite tuples, and $A$ a set. Then $a$ and $b$ are said to have the same Lascar strong type over $A, \operatorname{Lstp}(a / A)=\operatorname{Lstp}(b / A)$, if whenever $E(x, y)$ is an equivalence relation with a small number of classes which is defined by a partial type over $A$, we have $E(a, b)$.

The Independence Theorem for Lascar strong types is:

Fact 2.10 ( $T$ simple). Suppose $A \subseteq B$ and $A \subseteq C$ with $B$ independent from $C$ over $A$. Suppose $a_{1}, a_{2}$ are tuples such that $a_{1}$ is independent from $B$ over $A, a_{2}$ is independent from $C$ over $A$, and $\operatorname{Lstp}\left(a_{1} / A\right)=\operatorname{Lstp}\left(a_{2} / A\right)$. Then there is a such that $\operatorname{tp}(a / B)=\operatorname{tp}\left(a_{1} / B\right), \operatorname{tp}(a / C)=t p\left(a_{2} / C\right), a$ is independent from $B \cup C$ over $A$, and $\operatorname{Lstp}(a / A)=\operatorname{Lstp}\left(a_{1} / A\right)$.

Here is a convenient reformulation:

Fact 2.11. Let $A$ be a set. Let a be independent from $b$ over $A$, and suppose that $\Sigma(x, a) \cup \Gamma(x, b)$ does not fork over $A$. Let $a^{\prime}$ be such that $\operatorname{Lstp}(a / A)=\operatorname{Lstp}\left(a^{\prime} / A\right)$ and $a^{\prime}$ is independent from $b$ over $A$. Then $\Sigma\left(x, a^{\prime}\right) \cup \Gamma(x, b)$ does not fork over $A$.

Proof. Let $c$ realise $\Sigma(x, a) \cup \Gamma(x, b)$ such that $\operatorname{tp}(c / A, a, b)$ does not fork over $A$. (Thus $\{a, b, c\}$ is $A$-independent.) So $a$ is independent from $c$ over $A, a^{\prime}$ is independent from $b$ over $A$, and $L \operatorname{stp}(a / A)=\operatorname{Lstp}\left(a^{\prime} / A\right)$. By Fact 2.10, we can find $a^{\prime \prime}$ such that $t p\left(a^{\prime \prime} / A b\right)=t p\left(a^{\prime} / A b\right), t p\left(a^{\prime \prime} / A c\right)=t p(a / A c)$, and $\left\{a^{\prime \prime}, b, c\right\}$ is $A$-independent. Let $f$ be an $A$-automorphism fixing $b$ and taking $a^{\prime \prime}$ to $a^{\prime} . f(c)$ solves the problem.

Definition 2.12. (i) $T$ is supersimple if any complete type $p(x) \in S(B)$, where $x$ is a finite tuple of variables, does not fork over some finite subset $A$ of $B$.

(ii) ( $T$ simple.) The (ordinal or $\infty$-valued) $S U$ rank on complete types is defined by: $S U(p) \geq 0$ for all $p, S U(p) \geq \alpha+1$ if $p$ has a forking extension $q$ with $S U(q) \geq \alpha$, and for limit ordinal $\delta, S U(p) \geq \delta$ iff $S U(p) \geq \alpha$ for all $\alpha<\delta$. (We sometimes write $S U(a / A)$ for $S U(t p(a / A))$.) 
(iii) ( $T$ simple.) The ordinal or $\infty$-valued $D$ rank is defined on formulas by $D(\phi(x)) \geq 0$ if $\phi(x)$ is consistent, $D(\phi(x)) \geq \alpha+1$ if, for some set $A$ over which $\phi(x)$ is defined, there is a formula $\psi(x)$ which implies $\phi(x)$ and forks over $A$. As in (i) for limit $\delta$. For $\Sigma(x)$ a partial type closed under conjunctions $D(\Sigma)=\min \{D(\phi(x)$ : $\phi \in \Sigma\}$.

Fact 2.13. The following are equivalent:

(i) $T$ is supersimple.

(ii) $T$ is simple and for all complete types $p(x)$ in finitely many variables, $S U(p)<$ $\infty$.

(iii) $T$ is simple and for each formula $\phi(x), D(\phi(x))<\infty$.

Remark 2.14. Suppose $T$ is supersimple. Let $a$ be a possibly infinite tuple, and $A$ a set. Then $S U(t p(a / A))<\infty$ iff there is a finite subtuple $a^{\prime}$ of $a$ such that $a \subseteq \operatorname{acl}\left(A, a^{\prime}\right)$.

Now we pass to hyperimaginaries. By a type-definable equivalence relation over $A$ we mean an equivalence relation $E(x, y)$ on possibly infinite tuples (but of bounded length), which is defined by a partial type $\Phi(x, y)$ with parameters from $A$. By a hyperimaginary we mean an object of the form $a / E$ (i.e. the class of $a \bmod E$ ) where $E$ is type-definable over $\emptyset$. It is not in general possible to formally adjoin sorts $S_{E}$ for hyperimaginaries, and obtain a saturated (many-sorted) first order structure. Nevertheless, much of the machinery of model theory applies to hyperimaginaries. Note that everything we have dealt with so far is a hyperimaginary (real elements, imaginary elements, and even infinite tuples of imaginaries). Note that $A u t(\bar{M})$ acts on everybody. If $d, e$ are hyperimaginaries, then we say $e \in d c l(d)$ if $\operatorname{Aut}_{d}(\bar{M}) \subseteq \operatorname{Aut}_{e}(\bar{M})$, and $e \in b d d(d)$ if $e$ has a bounded number of images under $A u t_{d}(\bar{M})$. Note that if $e$ is an imaginary element, then $e \in b d d(d)$ iff $e \in \operatorname{acl}(d)$ (namely has only finitely many images under $d$ automorphisms). We will say that hyperimaginaries $e, d$ are equivalent (or interdefinable) if $d c l(e)=d c l(d)$. Similarly for interbounded.

Definition 2.15. We say that $T$ has elimination of hyperimaginaries if any hyperimaginary $e$ is equivalent to a tuple of elements of $\bar{M}^{e q}$.

The main point of this paper is to prove that supersimple theories eliminate hyperimaginaries.

What is crucial is that we can talk about $t p(e / d)$ if $e, d$ are hyperimaginaries: Suppose that $e=a / E$ and $d=b / F$. Let $\phi(x, y)$ be an $L$-formula. Let $\phi_{E, F}(x, y)$ be the partial type " $\exists x^{\prime}, y^{\prime}\left(E\left(x, x^{\prime}\right) \wedge F\left(y, y^{\prime}\right) \wedge \phi\left(x^{\prime}, y^{\prime}\right)\right)$ ". Actually the above is an infinitary formula but by compactness (saturation) is equivalent to the set of its "approximations" which is a partial type. We define $t p(e / d)$ to be the union of all those partial types $\phi_{E, F}(x, b)$ such that $\models \phi_{E, F}(a, b)$. Although as a set of formulas this has parameter $b$, a different choice of $b^{\prime}$ ( $F$-equivalent to $b$ ) would yield an equivalent partial type. Similarly we can define equality of Lascar strong types. Let $S_{E}$ be the "sort" consisting of the E-classes. Consider partial types $\Phi(x, y)$ which are $d$-invariant and induce an equivalence relation on $S_{E}$ with a bounded number of classes. We say $\operatorname{Lstp}((a / E) / d)=\operatorname{Lstp}\left(\left(a^{\prime} / E\right) / d\right)$ if $\Phi\left(a, a^{\prime}\right)$ holds for all such $\Phi$.

We have:

Fact 2.16. $t p(e / d)=t p\left(e^{\prime} / d\right)$ iff there is an automorphism of $\bar{M}$ which fixes $d$ and takes $e$ to $e^{\prime}$. 
See 2.19 (ii) for a similar statement for Lascar strong types.

The following result, originating in [13] will be useful:

Lemma 2.17. Suppose that a is a tuple from $\bar{M}^{e q}$, and e is a hyperimaginary such that $e \in d c l(a)$ and $a \in b d d(e)$. Then $e$ is equivalent to a tuple from $\bar{M}^{e q}$.

Proof. Suppose $a=\left(a_{i}: i \in I\right)$. For each finite $J \subseteq I$, let $a_{J}=\left(a_{i}: i \in J\right)$. So each such $a_{J} \in \operatorname{acl}(e)$. Let $b_{J}$ be the finite set of $e$ conjugates of $a_{J}$, viewed as a single element of $\bar{M}^{e q}$. So $b=\left(b_{J}: J\right.$ a finite subset of $\left.I\right)$ is a sequence of imaginaries. Clearly $b \in d c l(e)$. We want to see that $e \in d c l(b)$. Let $f$ be an automorphism fixing $b$, so fixing each $b_{J}$. It follows by definition of $b_{J}$ that $t p\left(a_{J} / e\right)=\operatorname{tp}\left(f\left(a_{J}\right) / e\right)$ (for each finite $J \subseteq I$ ), and thus $t p(a / e)=t p(f(a) / e)$. As $e \in d c l(a), f$ has to fix $e$. So $b$ is equivalent to $e$.

The next result is new.

Lemma 2.18. Let $e, d$ be hyperimaginaries and suppose $e \in b d d(d)$. Then the set of $d$ conjugates of $e$ is equivalent to a hyperimaginary $e^{\prime}$. (That is, an automorphism $f$ fixes setwise the set of $d$-conjugates of e iff $f\left(e^{\prime}\right)=e^{\prime}$.)

Proof. Let $e=a / E$, where $a$ is a tuple from $\bar{M}^{e q}$. For each (symmetric) formula $\phi(x, y) \in E(x, y)$, let $a_{\phi}^{0}, \ldots, a_{\phi}^{n_{\phi}}$ be a maximal set of realisations of $t p(a / d)$ such that $=\neg \phi\left(a_{\phi}^{i}, a_{\phi}^{j}\right)$ for $i \neq j$. Let $r\left(z, x_{\phi}^{i}: \phi \in E, i \leq n_{\phi}\right)$ be the partial type saying $\operatorname{tp}\left(z, x_{\phi}^{i}\right)=\operatorname{tp}(d, a)$ for all $\phi, i$, and $\neg \phi\left(x_{\phi}^{i}, x_{\phi}^{j}\right)$ for $i \neq j$. Let $F\left(z_{1}, z_{2}\right)$ be the type-definable relation $\exists \bar{x}\left(r\left(z_{1}, \bar{x}\right) \wedge r\left(z_{2}, \bar{x}\right)\right)$ on $\operatorname{tp}(d) \times t p(d)$. It is easy to see that for any automorphism $f$ of $\bar{M}, F(d, f(d))$ holds iff $f$ fixes setwise the set of $d$-conjugates of $e$. So $F$ is a type-definable equivalence relation on $t p(d)$, and $d / F$ is equivalent to the set of $d$-conjugates of $e$.

It is important to note that if $d, e, f$ are hyperimaginaries, then the notion "tp $(e / d)$ divides over $f$ " is well defined.

Fact 2.19. Suppose $T$ is simple.

(i) Remark 2.3 (ii) and Facts 2.5, 2.7, 2.10 and 2.11 hold for hyperimaginaries in place of tuples from $\bar{M}^{e q}$.

(ii) For $e, d, f$ hyperimaginaries, $\operatorname{Lstp}(d / f)=\operatorname{Lstp}(e / f)$ iff $t p(d / b d d(f))=$ $t p(e / b d d(f))$.

(iii) Suppose $T$ is supersimple, and suppose e is a hyperimaginary element which is in $b d d(a A)$ for a some finite tuple from $\bar{M}^{e q}$. Then $S U(\operatorname{tp}(e / A))<\infty$.

From now on, $a, b, e, \ldots$ will range over hyperimaginaries, unless we say otherwise. Note that a set $A$ of parameters will, under a given enumeration, also be a hyperimaginary, but we stick with notation $A, B, \ldots$ for sets of parameters.

Definition 2.20 ( $T$ simple). By an amalgamation base, we mean a complete type $p(x)$ over a set $A$ (possibly of hyperimaginaries) such that the Independence Theorem holds for $p$ over $A$. Namely, whenever $B, C \subseteq A$ are independent over $A$ and $p_{1}(x), p_{2}(x)$ are complete nonforking extensions of $p$ over $B, C$ respectively, then $p_{1}(x) \cup p_{2}(x)$ extends to a nonforking extension $q(x) \in S(B \cup C)$ of $p$ (equivalently does not fork over $A$ ).

Note that any Lascar strong type (complete type over a $b d d$-closed set by 2.17 (ii)) is an amalgamation base. 
Definition 2.21 ( $T$ simple). Let $p(x) \in S(A)$ be an amalgamation base. Then the canonical base of $p, C b(p)$, is defined to be the smallest (up to equivalence) hyperimaginary $e \in \operatorname{dcl}(A)$ such that $p$ does not fork over $e$ and $p \mid e$ is an amalgamation base.

Fact 2.22 ( $T$ simple). Canonical bases exist. Moreover $C b(p)$ has the following alternative description: Let $\mathbf{P}_{p}$ be the family of amalgamation bases $q(x)$ for which there are amalgamation bases $p=p_{0}, p_{1}, \ldots, p_{k}=q$ (some $k$ ) such that $p_{i}$ and $p_{i+1}$ have a common nonforking extension. Then an automorphism $f$ of $\bar{M}$ fixes $C b(p)$ pointwise iff it fixes $\mathbf{P}_{p}$ setwise. In particular $C b(p)=C b(q)$ whenever $q \in \mathbf{P}_{p}$.

Remark 2.23. $T$ is stable just if $T$ is simple and for any complete type $p(x)$ over a model $M$ (elementary substructure of $\bar{M}$ ), $p$ has a unique nonforking extension over any $B \supset M$. If $T$ is stable, then any type over a model is definable (for each $\phi(x, y) \in L$ there is $\psi(y)$ over $M$ such that $\phi(x, b) \in p(x)$ iff $\models \psi(b)$ for all $b \in M)$. In this (stable) case, $C b(p)$ is precisely the sequence of these defining formulas, considered as elements of $\bar{M}^{e q}$.

Fact 2.24 ( $T$ simple). Let $p(x) \in S(A)$ be an amalgamation base. Let $\left(a_{i}: i<\omega\right)$ be a Morley sequence in $p$. Then $C b(p) \in d c l\left(a_{i}: i<\omega\right)$. Moreover if $T$ is supersimple and $S U(p)<\infty$, then $C b(p) \in d c l\left(a_{i}: i<k\right)$ for some $k<\omega$.

Proof. We will prove Fact 2.24 assuming that $x$ is a finite tuple from $\bar{M}^{e q}$. Let $a$ be such that $\left(a_{i}: i<\omega\right) \frown(a)$ is indiscernible. So $q(x)=\operatorname{tp}\left(a / A \cup\left\{a_{i}: i<\right.\right.$ $\omega\}$ ) is a nonforking extension of $p(x)$. But clearly $q(x)$ is finitely satisfiable in $\left(a_{i}: i<\omega\right)$ so does not fork over $\left(a_{i}: i<\omega\right)$. Let $r(x)=\operatorname{tp}\left(a /\left(a_{i}: i<\omega\right)\right)$. We claim that $r(x)$ implies $\operatorname{Lstp}\left(a /\left(a_{i}: i<\omega\right)\right)$ : Let $E(x, y)$ be a type-definable equivalence relation over $\left(a_{i}: i<\omega\right)$ with a small number of classes. Let $\phi(x, y)$ be a formula in $E(x, y) . \phi(x, y)$ has parameters from $a_{0}, \ldots, a_{n}$, say. As there do not exist infinitely many tuples, each distinct pair from which satisfies $\neg \phi(x, y)$, it follows from indiscernibility that $\phi\left(a_{i}, a\right)$ holds for all $i>n$. It easily follows (as $E$ is an equivalence relation) that $E(a, b)$ for any other realisation $b$ of $r(x)$. Thus $r(x)$ implies $\operatorname{Lstp}\left(a /\left(a_{i}: i<\omega\right)\right)$ as claimed, and so, by the Independence Theorem, $r(x)$ is an amalgamation base. Thus $C b(p)=C b(r) \in \operatorname{dcl}\left(a_{i}: i<\omega\right)$ as required.

Finally, we make a rather useful observation on type-definable equivalence relations.

Lemma 2.25 ( $T$ supersimple). Let a be a possibly infinite tuple of elements from $\bar{M}^{e q}$. Let $q(x)=t p(a)$. Assume $S U(q)<\infty$. Let $E$ be a type-definable (over $\emptyset)$ equivalence relation on $q$. Then there is a formula $\psi(x, y) \in E(x, y)$ such that whenever $b, c$ realise $q$ and $=\psi(b, c)$, then $b / E$ and $c / E$ are interbounded.

Proof. As $S U(q)<\infty, a \in \operatorname{acl}\left(a^{\prime}\right)$ for a suitable finite subtuple $a^{\prime}$ of $a . x^{\prime}$ denotes the corresponding finite subtuple of $x$. Among formulas $\theta\left(x^{\prime}, a\right) \in E(x, a)$ choose one of least $D$-rank. Call it $\theta\left(x^{\prime}, a\right)$ and suppose its $D$-rank is $\alpha$. Note that $\alpha$ is smallest among the $D$-ranks of formulas $\phi\left(x^{\prime \prime}, a\right) \in E(x, a)$ for $x^{\prime \prime}$ a finite subtuple of $x$ containing $x^{\prime}$.

Now, by compactness, find a symmetric formula $\theta_{1}(x, y) \in E(x, y)$ implying $\theta$ and such that

$$
\models \theta_{1}(x, y) \wedge \theta_{1}(y, z) \rightarrow \theta(x, z)
$$


Let $\psi(x, y) \in E(x, y)$ be symmetric such that

$$
\models \psi(x, y) \wedge \psi(y, z) \wedge \psi(z, w) \rightarrow \theta_{1}(x, w),
$$

and suppose that $b$ realises $q$ and $\models \psi(a, b)$.

Claim. b/E $\in$ bdd $(a / E)$.

Proof of claim. Suppose not. Then we can find an infinite indiscernible sequence $\left(b_{i}: i<\omega\right)$ of realisations of $t p(b /(a / E))$ such that $\neg E\left(b_{i}, b_{j}\right)$ for $i \neq j$. In fact by indiscernibility there is a formula $\chi(x, y) \in E(x, y)$ such that $\neg \chi\left(b_{i}, b_{j}\right)$ for each $i \neq j$. Without loss of generality, $\psi\left(a, b_{0}\right)$. For each $i$ there is $a_{i}$ such that $E\left(a, a_{i}\right)$ and $\psi\left(a_{i}, b_{i}\right)$. So by the choice of $\psi$ each $b_{i}$ satisfies $\theta_{1}(x, a)$.

Let $\phi(x, y) \in E(x, y)$ be symmetric, such that

$$
\models \phi(x, y) \rightarrow \theta_{1}(x, y) \quad \text { and } \quad \models \phi(x, y) \wedge \phi(y, z) \rightarrow \chi(x, z) .
$$

It follows that

(i) for each $b_{i}, \phi\left(x, b_{i}\right) \rightarrow \theta(x, a)$, and

(ii) $\phi\left(x, b_{i}\right) \wedge \phi\left(x, b_{j}\right)$ is inconsistent for $i \neq j$.

We may rechoose the $b_{i}$ such that they still each realise $q$, (i), (ii) remain true and $\left(b_{i}: i<\omega\right)$ is indiscernible over $a$. But then $\phi\left(x, b_{0}\right)$ forks over $a$, and so by (i) $D\left(\phi\left(x, b_{0}\right)<D(\theta(x, a))\right.$, contradicting the choice of $\theta$.

The Claim is proved. By symmetry, $a / E \in b d d(b / E)$ too, and Lemma 2.25 is proved.

\section{The BASIC ARGUMEnt}

We assume $T$ to be supersimple in this section.

Let $a$ be a finite tuple of real elements, and $E$ a type-definable equivalence relation over $\emptyset$ on $q=t p(a)$. Let us assume that $t p(a /(a / E)) \models \operatorname{Lstp}(a /(a / E))$.

By Lemma 2.25, let $\psi_{0}(x, y)$ be a formula in $E$ such that for $a, b$ realising $q$, if $\psi_{0}(a, b)$ then $a / E$ and $b / E$ are interbounded. Let $\psi_{1}(x, y) \in E$ be such that if $b, c, c^{\prime}$ realise $q, E\left(c^{\prime}, c\right)$ and $\exists x\left(\psi_{1}(x, c) \wedge \psi_{1}(x, b)\right)$ then $\psi_{0}\left(c^{\prime}, b\right)$ (by compactness). Let $\Psi(x, y)$ be the set of formulas in $E$ which imply $\psi_{1}(x, y)$. With the above notation, we make:

Definition 3.1. Let $\psi(x, y) \in \Psi(x, y)$, and let $a, b$ realise $q$. We define $S_{\psi}(a, b)$ to hold if there exists $c$ realising $q$ such that

(i) $E(c, a)$, and $t p(c / b,(a / E))$ does not fork over $(a / E)$,

(ii) $\psi_{0}(c, b)$ holds, and $\psi(x, c) \wedge \psi(x, b)$ does not fork over $a / E$.

Remark 3.2. (i) If $E\left(a, a^{\prime}\right)$, then $S_{\psi}(a, b)$ iff $S_{\psi}\left(a^{\prime}, b\right)$.

(ii) $E(a, b)$ iff $S_{\psi}(a, b)$ holds for all $\psi \in \Psi$.

Proof. (i) is immediate from the definition of $S_{\psi}$.

(ii) Left to right follows from the Independence Theorem for Lascar strong types. Now assume the right hand side. So for each $\psi \in \Psi$, there is $c$ such that $\models E(c, a)$ and $\psi(x, c) \wedge \psi(x, b)$ is consistent. As $\Psi$ axiomatizes $E$, there is, by compactness, $c$ such that $\models E(c, a)$ and $E(x, c) \wedge E(x, b)$ is consistent. Thus $E(a, b)$.

Remark 3.3. Suppose for each $\psi \in \Psi, S_{\psi}$ is definable $($ on $q \times q)$. Then $E$ is equivalent on $q$ to a conjunction of definable equivalence relations. 
Proof. Fix $\psi$, and suppose $\rho_{\psi}(x, y)$ is a formula defining $S_{\psi}$ on $q \times q$. By Remark $3.2(\mathrm{i})$ and compactness there is a formula $\pi(x) \in q$ such that

$$
E(x, y) \models \forall z\left(\pi(z) \rightarrow\left(\rho_{\psi}(x, z) \leftrightarrow \rho_{\psi}(y, z)\right)\right) .
$$

Let $E_{\psi}(x, y)$ be the formula on the right hand side, which defines an equivalence relation. Suppose that $E_{\psi}(a, b)$ holds for all $\psi \in \Psi(a, b$ realising $q)$. As $\rho_{\psi}(a, a)$ holds for all $\psi$ it follows that $\rho_{\psi}(a, b)$ holds for all $\psi$, so, by Remark 3.2, $E(a, b)$ holds. Thus $E$ is equivalent on $q \times q$ to the conjunction of the $E_{\psi}$.

Thus the main issue is the definability of the relations $S_{\psi}$.

Lemma 3.4. $S_{\psi}$ is type-definable $($ on $q \times q)$.

Proof. For any $L$-formula $\phi(x, w)$ and $k<\omega$, let $n_{\phi, k}=D(E(x, a), \phi, k)$. Then $a, c, b$ satisfy conditions (i) and (ii) of Definition 3.1 iff $a, b, c$ realise $q, E(a, c)$, $D(t p(c / b,(a / E)), \phi, k) \geq n_{\phi, k}$ for all $\phi, k, \psi_{0}(c, b)$ and

$(*)$ there is a Morley sequence $\left(\left(c_{i}, b_{i}\right): i<\omega\right)$ in $t p(c, b /(a / E))$ such that $\left\{\psi\left(x, c_{i}\right) \wedge \psi\left(x, b_{i}\right): i<\omega\right\}$ is consistent.

Indiscernibility over $a / E$ of the sequence $\left(c_{i}, b_{i}\right)_{i}$ is expressible. As for the independence: note that, as $\psi_{0}(c, b), a / E$ and $b / E$ are interbounded. So independence of $\left(c_{i}, b_{i}\right)_{i}$ over $a / E$ is equivalent to $\left(b_{i}\right)_{i}$ is $b / E$-independent, and for each $i, c_{i}$ is independent from $\left(c_{j}: j<i\right) \cup\left(b_{j}: j<\omega\right)$ over $a / E$. These are again expressible using the $D(-, \phi, k)$. (See Fact 2.5(ii).)

We now see what can be said regarding the type-definability of $\neg S_{\psi}(x, y)$.

Lemma 3.5. Let $a, b$ realise $q$, and let $\psi \in \Psi$. Then $\neg S_{\psi}(a, b)$ holds iff there is $c$ realising $q$ such that

(i) $E(c, a)$ and $t p(c / b,(a / E))$ does not fork over a/E, and

(ii) either $\neg \psi_{0}(c, b)$ or $\psi(x, c) \wedge \psi(x, b)$ forks over $a / E$.

Proof. Left to right is immediate. For suppose that $\neg S_{\psi}(a, b)$. Choose $c$ satisfying (i). Then from Definition 3.1 we see that (ii) in the lemma holds.

Conversely, suppose $S_{\psi}(a, b)$, witnessed by $c^{\prime}$ as in Definition 3.1. Now choose any $c$ satisfying (i) in the lemma. We want to show that (ii) fails. By Fact 2.11 $\left(\right.$ as $\left.\operatorname{Lstp}(c /(a / E))=\operatorname{Lstp}\left(c^{\prime} /(a / E)\right)\right), \psi(x, c) \wedge \psi(x, b)$ does not fork over $a / E$, so in particular is consistent. As $\models \psi \rightarrow \psi_{1}$ we see from the choice of $\psi_{1}(x, y)$ that $\psi_{0}(c, b)$.

Lemma 3.6. Let $\psi(x, y) \in \Psi(x, y)$. Suppose that there is some $k$ such that whenever $c, b$ realise $q, c / E, b / E$ are interbounded, $c$ is independent from $b$ over $c / E$, and $\psi(x, c) \wedge \psi(x, b)$ forks over $c / E$, then for any $c / E$-independent sequence $\left(\left(c_{i}, b_{i}\right)\right.$ : $i<k)$ of realisations of $t p(c, b /(c / E)),\left\{\psi\left(x, c_{i}\right) \wedge \psi\left(x, b_{i}\right): i<k\right\}$ is inconsistent. Then $S_{\psi}(x, y)$ is definable $($ on $q \times q)$.

Proof. Given the assumptions of the lemma, we see from Lemma 3.5 that $\neg S_{\psi}(x, y)$ is type-definable: (i) is clear and (ii) says if $\psi_{0}(c, b)$ then $\psi(x, c) \wedge \psi(x, c)$ forks over $a / E$. The latter is, by the assumptions, equivalent to the existence of a Morley sequence $\left(c_{i}, b_{i}\right)_{i<\omega}$ in $t p(c, b /(a / E))$ such that $\left\{\psi\left(x, c_{i}\right) \wedge \psi\left(x, b_{i}\right): i<k\right\}$ is inconsistent.

Together with Lemma 3.4 and compactness, we see that $S_{\psi}$ is definable. 
Remark 3.7. (i) In a low theory, the assumptions of Lemma 3.6 hold for all $\psi$. Thus for supersimple low theories, we have proved that $E$ is equivalent to a conjunction of definable equivalence relations. In fact the reader should note that our proof also yields the equality of Lascar strong type with strong type in low simple theories.

(ii) In the general supersimple case, our proof in section 5 will more or less reduce the situation to one where the assumptions of Lemma 3.6 do hold.

Remark 3.8. The discussion above assumed that $t p(a /(a / E)) \models \operatorname{Lstp}(a /(a / E))$. We can however always reduce to this case, using 2.17. Given arbitrary typedefinable $E$ on $q$, let $a$ realise $q$ and let $e=d c l(a) \cap b d d(a / E)$. Then $t p(a / e) \models$ $\operatorname{Lstp}(a / e) \models \operatorname{Lstp}(a /(a / E))$ and $e=a / E^{\prime}$ for some type-definable $E^{\prime}$ refining $E$ on $q$. Note that $a / E \in \operatorname{dcl}\left(a / E^{\prime}\right)$ and $a / E^{\prime} \in b d d(a / E)$. If we have proved that $E^{\prime}$ is equivalent on $q$ to a conjunction of definable equivalence relations, then, by 2.17 , the same is true of $E$.

\section{Analysability}

The proof of our main theorem (elimination of hyperimaginaries in supersimple theories) will need some more machinery, which we develop in this section.

Definition 4.1 ( $T$ simple). Let $A$ be a set and $p(x)$ a complete type over $A$. Let $\Sigma$ be a family of partial types which is invariant under $A_{u t}(\bar{M})$. We say that $p(x)$ is $\Sigma$-internal if there is some $B \supseteq A$ and a sequence $\left(b_{i}: i<\alpha\right)$ of realisations of types in $\Sigma$ which are over $B$, and there is some realisation $a$ of $p(x)$ such that $a$ is independent from $B$ over $A$ and $a \in \operatorname{dcl}\left(B, b_{i}: i<\alpha\right)$.

Recall that (for $T$ simple), complete types $p(x), q(y)$ are orthogonal if whenever $p^{\prime}(x), q^{\prime}(y)$ are nonforking extensions of $p, q$ respectively over a common domain $C$, then for any realisations $a$ of $p^{\prime}$ and $b$ of $q^{\prime}, a$ is independent from $b$ over $C$.

Lemma 4.2 (T simple). Suppose tp $(a / A)$ is nonorthogonal to some type $q$. Then there is $a_{0} \in \operatorname{dcl}(a, A) \backslash b d d(A)$ such that $\operatorname{tp}\left(a_{0} / A\right)$ is internal in the family of $A$-conjugates of $q$.

Proof. The situation allows us to assume that $q$ is over a set $B_{0}$ where $a$ is independent from $B_{0}$ over $A$ and moreover there is a realisation $b_{0}$ of $q$ such that $t p\left(a / B_{0}, b_{0}\right)$ forks over $B_{0}$. Let $\left(B_{i}, a_{i}: i<\omega\right)$ be a Morley sequence in $\operatorname{tp}\left(B_{0}, b_{0} / A, a\right)$. Let $e$ $=C b\left(\left(B_{0}, b_{0} / b d d(A, a)\right)\right)$. By Fact 2.24, $e \in d c l\left(B_{i}, b_{i}: i<\omega\right)$. Also $e \in b d d(A, a)$. On the other hand $e \notin b d d(A)$ (for otherwise $\left(B_{0}, b_{0}\right)$ would be independent from $a$ over $A)$. Note that $a$ and thus also $e$ is independent from $\left(B_{i}: i<\omega\right)$ over $A$. Thus (as $b_{i}$ realises a conjugate of $q$ over $B_{i}$ ), $e$ is internal in the family of $A$-conjugates of $q$. Remember that $e \in \operatorname{bdd}(A, a)$. By Lemma 2.18, let $a_{0}$ be a hyperimaginary equivalent to the set of $(A, a)$ conjugates of $e$. Then $t p\left(a_{0} / A\right)$ is still internal in the family of $A$-conjugates of $q$ and $a_{0} \notin b d d(A)$.

Now we introduce a certain "analysability rank" for supersimple theories, which will be crucial for the proof of our main result in section 5 .

For the remainder of this section we assume $T$ to be supersimple.

Lemma 4.3. Let $S U(a / A)<\infty$. Then there are (hyperimaginaries) $a_{0}, a_{1}, \ldots, a_{n} \in$ $\operatorname{dcl}(A, a)$ (some $n<\omega)$ and ordinals $\alpha_{i}$ for $i<n$ such that $a_{0}=($ some enumeration of ) $A, a \in b d d\left(a_{n}\right)$ and for each $i<n, a_{i} \in d c l\left(a_{i+1}\right), t p\left(a_{i+1} / a_{i}\right)$ is internal in a family of formulas of $D$-rank $\alpha_{i}$, and $t p\left(a / a_{i}\right)$ is orthogonal to all types of $D$-rank $<\alpha_{i}$. 
Proof. Put $a_{0}$ to be some enumeration of $A$. If $S U(a / A)=0$, then $a \in b d d\left(a_{0}\right)$ and we are finished. Suppose otherwise. It is clear that there is some $b \in \bar{M}^{e q}$ such that $a$ forks with $b$ over $A$. For any such $b, D(t p(b / A))<\infty$, so let $\alpha_{0}$ be the least ordinal such that $\operatorname{tp}(a / A)$ is nonorthogonal to some type of $D$-rank $\alpha_{0}$. By Lemma 4.2 , there is $a_{1} \in d c l(A, a) \backslash b d d(A)$ such that $t p\left(a_{1} / a_{0}\right)$ is internal in the family of $A$-conjugates of $q$, so in particular internal in the family of formulas of $D$-rank $\alpha_{0}$. As $a$ forks with $a_{1}$ over $A, S U\left(a / A, a_{1}\right)<S U(a / A)$, so continuing we finish after finitely many steps.

Definition 4.4. Given $a$ and $A$ such that $S U(a / A)<\infty$, we define $R(a / A)(=$ $R(t p(a / A)))$ to be the least ordinal $\alpha$ such that for some $n<\omega$ there are $a_{0}, \ldots, a_{n}$ and ordinals $\alpha_{0}, \ldots, \alpha_{n-1}$ as in Lemma 4.3, with $\alpha_{i} \leq \alpha$ for all $i<n$.

Lemma 4.5. Suppose tp $(a / A)$ is internal in an A-invariant family $\Sigma$ of partial types and $a^{\prime} \in \operatorname{dcl}(A a)$. Then $t p\left(a^{\prime} / B\right)$ is $\Sigma$-internal for any $B \supset A$.

Proof. For some $C \supset A$ such that $a$ is independent from $C$ over $A, a \in \operatorname{dcl}(b)$ where $b$ is a tuple of realisations of types in $\Sigma$ which are over $C$. Let $C^{\prime}$ realise a nonforking extension of $\operatorname{tp}(C / A, a)$ over $(B, a)$. Then $a$ is independent from $C^{\prime}$ over $B$ and $a \in d c l\left(b^{\prime}, A\right)$ where $b^{\prime}$ is the image of $b$ under some $(A, a)$-automorphism taking $C$ to $C^{\prime}$. The same thing is true for $a^{\prime}$ in place of $a$. As $\Sigma$ is $A$-invariant, $\operatorname{tp}\left(a^{\prime} / B\right)$ is internal to $\Sigma$.

Corollary 4.6. (i) Suppose $a=\left(e_{i}: i \in I\right)$. Suppose $S U(a / A)<\infty$ and $R\left(e_{i} / A\right)<$ $\alpha$ for each $i$. Then $R(a / A)<\alpha$.

(ii) Suppose $b \in \operatorname{dcl}(a A)$. Then $R(b / A) \leq R(a / A)$.

Proof. (i) By Lemma 4.5, every nonbounded extension of $t p(a / A)$ is nonorthogonal to a type of $D$-rank $<\alpha$. Thus we can build an analysis of $t p(a / A)$ in formulas of $D$-rank less than $\alpha$, taking at each step the formulas of minimal $D$-rank possible to which $t p(a / B)$ is nonorthogonal. As $S U(a / A)<\infty$, this analysis is finite, whereby, $R(a / A)<\alpha$.

(ii) Similar.

Lemma 4.7. Suppose $S U(a / A)<\infty$. Let $a^{\prime}$ be some enumeration of $\{b \in d c l(A, a)$ : $R(b / A)<\alpha\}$. Then $R\left(a^{\prime} / A\right)<\alpha$ and $t p\left(a / a^{\prime}\right)$ is orthogonal to all formulas of $D$ rank $<\alpha$.

Proof. $R\left(a^{\prime} / A\right)<\alpha$ by Corollary 4.6 .

Suppose for a contradiction that $t p\left(a / a^{\prime}\right)$ is nonorthogonal to a formula of $D$ rank $<\alpha$. By 4.2 there is $c \in d c l\left(a, a^{\prime}\right) \backslash b d d\left(a^{\prime}\right)$ such that $t p\left(c / a^{\prime}\right)$ is internal in some family of formulas of $D$-rank $\beta$ for some $\beta<\alpha$. Let $a_{0}=A, a_{1}, \ldots, a_{n}$ witness $R\left(a^{\prime} / A\right)<\alpha$. In particular $a^{\prime} \in b d d\left(a_{n}\right)$. So easily $t p\left(c / a_{n}\right)$ is internal in the family of formulas of $D$-rank $\beta$. It follows that $R\left(c, a_{n} / A\right)<\alpha$. As $\left(c, a_{n}\right) \in \operatorname{dcl}(a, A)$ it follows that $c \in \operatorname{dcl}\left(a^{\prime}\right)$, a contradiction.

\section{THE GENERAL CASE}

We assume $T$ to be supersimple. We will prove:

Theorem 5.1. Let e be a finitary hyperimaginary. Then e is interdefinable with a sequence of imaginaries (elements of $\bar{M}^{e q}$ ).

The theorem will be proved by an inductive argument and so we will prove the following stronger result. 
Proposition 5.2. Suppose a is a possibly infinite sequence of imaginary elements contained in the algebraic closure of a finite subtuple. Let $a_{0}$ be a hyperimaginary in $d c l(a)$. Then $a_{0}$ is interdefinable with a sequence of imaginaries.

The proposition will be proved by induction on $R\left(t p\left(a / a_{0}\right)\right)$.

Base step. $R\left(t p\left(a / a_{0}\right)\right)=0$. This means that $a \subseteq \operatorname{acl}\left(a_{0}\right)$, and is taken care of by Lemma 2.17.

We now want to prove the induction step. The key result enabling us to carry this out will be:

Lemma 5.3. Suppose that Proposition 5.2 holds for all a, $a_{0}$ such that $R\left(t p\left(a / a_{0}\right)\right)$ $<\alpha$. Now suppose (with notation as in Proposition 5.2) that $t p\left(a / a_{0}\right)$ is internal to formulas of D-rank $\alpha$ and is orthogonal to all types of D-rank strictly less than $\alpha$. Then $a_{0}$ is interdefinable with a sequence of imaginaries.

We will prove the lemma later. Assuming the lemma, we complete the proof of the proposition.

Induction step. Suppose the proposition holds whenever $R\left(t p\left(a / a_{0}\right)\right)<\alpha$. Then it holds whenever $R\left(t p\left(a / a_{0}\right)\right)=\alpha$.

Proof. Let $R\left(\operatorname{tp}\left(a / a_{0}\right)\right)=\alpha$. So there are hyperimaginaries $a_{1}, \ldots, a_{n} \in \operatorname{dcl}(a)$ such that $a \in b d d\left(a_{n}\right), a_{i} \in d c l\left(a_{i+1}\right), t p\left(a_{i+1} / a_{i}\right)$ is internal in formulas of $D$-rank $\alpha_{i}$, and $t p\left(a / a_{i}\right)$ is orthogonal to all formulas of $D$-rank $<\alpha_{i}$, where the $\alpha_{i} \leq \alpha(i<n)$. We proceed by induction on $n$. As $a_{n} \in \operatorname{dcl}(a)$ and $a \subseteq \operatorname{acl}\left(a_{n}\right)$ it follows by 2.17 that $a_{n}$ is equivalent to a sequence of imaginaries (also of ordinal valued $S U$-rank). So we assume $a_{n}$ to be a sequence of imaginaries. Now, either by hypothesis (if $\alpha_{n-1}<\alpha$ ) or by Lemma 5.3 (if $\alpha_{n-1}=\alpha$ ) we conclude that $a_{n-1}$ is equivalent to a sequence of imaginaries, and so we can assume $a_{n-1}$ to be a sequence of imaginaries. As $a_{0} \in \operatorname{dcl}\left(a_{n-1}\right)$, and $R\left(t p\left(a_{n-1} / a_{0}\right)\right) \leq \alpha$ (4.6 (ii)), we conclude by induction.

So, modulo Lemma 5.3, both Proposition 5.2 and Theorem 5.1 are proved.

Proof of Lemma 5.3. The proof is a rather fancy version of the basic argument of section 3 .

Note now that $a$ may be an infinite tuple. Write $a_{0}$ as $a / E$ for some $\emptyset$-definable equivalence relation $E$ on $q=t p(a)$. As in Remark 3.8 there is no harm in assuming that $t p(a /(a / E))$ implies $\operatorname{Lstp}(a /(a / E))$. Let $p_{a}(x)=t p(a /(a / E))(=E(x, a))$. By Lemma 2.25 , let $\psi_{0}(x, y) \in E(x, y)$ be such that $\psi_{0}(a, b)$ implies $a / E$ and $b / E$ are interbounded (for $a, b$ realising $q$ ). Let $\psi_{1}(x, y) \in E(x, y)$ be such that whenever $b, c, c_{1}$ realise $q$, and $\models E\left(c, c_{1}\right)$ and $\models \exists x\left(\psi_{1}(x, c) \wedge \psi_{1}(x, b)\right)$, then $\models \psi_{0}\left(c_{1}, b\right)$. Let $a^{\prime}$ be a finite subtuple of $a$ such that $a \in \operatorname{acl}\left(a^{\prime}\right)$. (Similarly for $x$ a tuple of variables representing $a, x^{\prime}$ will denote the corresponding finite subtuple.) $p_{a}^{\prime}\left(x^{\prime}\right)$ denotes $t p\left(a^{\prime} /(a / E)\right)$. Let $\Psi(x, y)$ be the set of formulas $\psi(x, y) \in E(x, y)$, which imply $\psi_{1}(x, y)$ and also imply that $x \subseteq \operatorname{acl}\left(x^{\prime}\right), y \subseteq \operatorname{acl}\left(y^{\prime}\right)$.

Note that $p_{a}^{\prime}\left(x^{\prime}\right)$ is a type in finitely many imaginary variables which is internal in formulas of $D$-rank $\alpha$ (and orthogonal to formulas of $D$-rank $<\alpha$ ). Let $A$ be a set of imaginary elements (a model for example) containing $a, \phi_{i}\left(z_{i}\right)$ formulas of $D$-rank $\alpha$ over $A$ for $i=1, \ldots, n, c_{i}$ realisations of $\phi_{i}$, and $d$ a realisation of $p_{a}(x)$ independent from $A$ over $a / E$ such that $d^{\prime} \in \operatorname{dcl}\left(A, c_{1}, \ldots, c_{n}\right)$. Let this be witnessed by an $A$-definable function $f$. (So $d^{\prime}=f\left(c_{1}, \ldots, c_{n}\right)$.) Let $\chi_{0}\left(x^{\prime}\right)$ be the formula $\exists z_{1}, \ldots, z_{n}\left(\phi_{1}\left(z_{1}\right) \wedge \ldots \wedge \phi_{n}\left(z_{n}\right) \wedge x^{\prime}=f\left(z_{1}, \ldots, z_{n}\right)\right)$. 
Note that only finitely many parameters from $A$ appear in $\chi_{0}$ (the parameters from the $\phi_{i}$ and from $f$ ). Let $\hat{a}$ be the sequence consisting of $a$ and these finitely many additional elements. Write $\chi_{0}\left(x^{\prime}, \hat{a}\right)$ for $\chi_{0}\left(x^{\prime}\right)$ to witness these parameters. Let $\hat{q}=t p(\hat{a})$, and let $p_{\hat{a}}(x)=t p(d / \hat{a})$ above.

Finally we want to define a certain equivalence relation $F$ on $\hat{q}$. Let $\hat{a}_{0}$ be the set of hyperimaginaries $e$ in $\operatorname{dcl}(\hat{a})$ such that $R(\operatorname{tp}(e /(a / E)))<\alpha$. So (some enumeration of) $\hat{a}_{0}$ is equivalent to $\hat{a} / F$ for some type-definable over $\emptyset$ equivalence relation $F$.

We record some facts regarding the very last construction.

Fact 5.4. (i) $\operatorname{tp}(\hat{a} /(\hat{a} / F))$ implies $\operatorname{Lstp}(\hat{a} /(\hat{a} / F))$.

(ii) $S U(t p(\hat{a}))<\infty$.

(iii) $a / E \in \operatorname{dcl}(\hat{a} / F)$.

(iv) $R(t p((\hat{a} / F) /(a / E)))<\alpha$.

(v) $\operatorname{tp}(\hat{a} /(\hat{a} / F))$ is orthogonal to all types of $D$-rank $<\alpha$.

Proof. (i) This follows because $b d d\left(\hat{a}_{0}\right) \cap d c l(\hat{a})=\hat{a}_{0}$.

(ii) $\hat{a}$ consists of the sequence $a$ plus finitely many other imaginaries.

(iii) is clear.

(iv) follows from (ii) and Lemma 4.7.

(v) is also clear by Lemma 4.7 .

We now define the relation $S_{\psi}$ for $\psi \in \Psi$. As a matter of notation, for $\hat{c}$ realising $\hat{q}, c$ and $c^{\prime}$ denote the tuples such that $\operatorname{tp}\left(\hat{c}, c, c^{\prime}\right)=\operatorname{tp}\left(\hat{a}, a, a^{\prime}\right)$

Definition 5.5. Let $\hat{a}$ realise $\hat{q}$ and $b$ realise $q$. Let $\psi(x, y) \in \Psi(x, y)$. Then $S_{\psi}(\hat{a}, b)$ holds if there is a realisation $\hat{c}$ of $\hat{q}$ such that

(i) $F(\hat{c}, \hat{a})$ and $\operatorname{tp}(\hat{c} / b,(\hat{a} / F))$ does not fork over $\hat{a} / F$.

(ii) $\psi_{0}(c, b)$ holds, and $\chi_{0}\left(x^{\prime}, \hat{c}\right) \wedge \psi(x, c) \wedge \psi(x, b)$ does not fork over $\hat{a} / F$.

Lemma 5.6. $S_{\psi}$ is type definable on $\hat{q} \times q$.

Proof. The only thing to worry about is to be able to express that $\left(\hat{c}_{i}, b_{i}\right)_{i}$ is a Morley sequence in $\operatorname{tp}((\hat{c}, b) /(\hat{a} / F))$, in particular that it is independent, given that (i) holds and $\psi_{0}(c, b)$ holds. By choice of $\psi_{0}, c / E(=a / E)$ and $b / E$ are interbounded. On the other hand, we are given that $t p(b /(b / E))$ is orthogonal to all formulas of $D$-rank $<\alpha$, and by Fact 5.4 (and interboundedness of $a / E$ and $b / E$ ), $R(t p((\hat{a} / F),(b / E)))<\alpha$. Hence $t p(b /(b / E))$ is orthogonal to $t p((\hat{a} / F) /(b / E))$. Thus a $\hat{a} / F$-indiscernible sequence $\left(b_{i}\right)_{i}$ of realisations of $t p(b /(\hat{a} / F))$ is $\hat{a} / F$ independent just if it is a $b / E$-independent sequence of realisations of $t p(b /(b / E))$. The latter is expressible using the $D(-, \phi, k)$ ranks as usual. It follows easily again using $D(-, \phi, k)$-ranks that for the $\left(\hat{c}_{i}, b_{i}\right)_{i}$ to be a Morley sequence over $\hat{a} / F$ is type-definable.

Lemma 5.7. $\neg S_{\psi}$ is also type-definable on $\hat{q} \times q$.

Proof. First, using the Independence Theorem (together with Fact 5.4 (i)), and choice of $\psi_{1}$ we can, as in Lemma 3.5, show that, for $\hat{a}$ realising $\hat{q}$ and $b$ realising $q$,

$(*) \neg S_{\psi}(\hat{a}, b)$ holds just if there is $\hat{c}$ realising $\hat{q}$ such that

(i) $F(\hat{c}, \hat{a})$ and $\operatorname{tp}(\hat{c} / b,(\hat{a} / F))$ does not fork over $\hat{a} / F$, and

(ii) either $\neg \psi_{0}(c, b)$ or $\chi_{0}\left(x^{\prime}, \hat{c}\right) \wedge \psi(x, c) \wedge \psi(x, b)$ forks over $\hat{a} / F$.

It is again the second clause of (ii) which poses the problem. 
Claim. Suppose (i) of (*) holds for $\hat{a}, \hat{c}, b$ and that $\psi_{0}(c, b)$ holds. Then $\chi_{0}\left(x^{\prime}, \hat{c}\right) \wedge$ $\psi(x, c) \wedge \psi(x, b)$ forks over $\hat{a} / F$ if and only if there is a Morley sequence $\left(\hat{c}_{i}, b_{i}\right)_{i}$ in $\operatorname{tp}((\hat{c}, b) /(\hat{a} / F))$ such that $\left\{\chi_{0}\left(x^{\prime}, \hat{c}_{i}\right) \wedge \psi\left(x, c_{i}\right) \wedge \psi\left(x, b_{i}\right): i=1, \ldots, n+2\right\}$ is inconsistent.

Proof. Right implies left is clear. We prove left implies right. Fix a Morley sequence $\left(\hat{c}_{i}, b_{i}\right)_{i}$ in $\operatorname{tp}((\hat{c}, b) /(\hat{a} / F))$. Suppose for the sake of contradiction that $e$ realises the finite set of formulas on the right hand side above. In particular for $1<i \leq n+2, e$ forks with $\left(\hat{c}_{i}, b_{i}\right)$ over $\hat{a} / F$. But $\left(\hat{c}_{i}, b_{i}\right)$ is independent from $\hat{c}_{1}$ over $\hat{a} / F$, whereby $\operatorname{tp}\left(e / \hat{c}_{i}, b_{i}, \hat{c}_{1}\right)$ forks over $\hat{c}_{1}$. As $\chi_{0}\left(e^{\prime}, \hat{c}_{1}\right)$ holds, there are formulas $\phi_{1}, \ldots, \phi_{n}$ over $\hat{c}_{1}$ of $D$-rank $\alpha$ and realisations $d_{i}$ of $\phi_{i}$ such that $e^{\prime} \in \operatorname{dcl}\left(\hat{c}_{1}, d_{1}, \ldots, d_{n}\right)$. It follows (as $\left.e \in \operatorname{acl}\left(e^{\prime}\right)\right)$ that $\left(d_{1}, \ldots, d_{n}\right)$ forks with $\left(\hat{c}_{i}, b_{i}\right)$ over $\hat{c}_{1}$ for $i=2, \ldots, n+2$.

Subclaim. $t p\left(\hat{c}_{i}, b_{i} / \hat{c}_{1}\right)$ is orthogonal to all types of $D$-rank $<\alpha$ for $i>1$.

Proof of subclaim. Clearly $\hat{c}_{i}$ is independent from $b_{i}$ over $\hat{c}_{1}$. Also $t p\left(\hat{c}_{i} / \hat{c}_{1}\right)$ being a nonforking extension of $\operatorname{tp}\left(\hat{c}_{i} /(\hat{a} / F)\right)$ is (by Fact $\left.5.4(\mathrm{v})\right)$ orthogonal to all types of $D$-rank $<\alpha$. Also $t p\left(b_{i} / b d d\left(\hat{c}_{1}\right)\right)$ does not fork over $b / E$ (see proof of Lemma 5.6) and so by assumption is orthogonal to all types of $D$-rank $<\alpha$. Thus the subclaim follows.

By the subclaim and the fact that $\left(\left(\hat{c}_{i}, b_{i}\right): i=2, \ldots, n+2\right)$ is $\hat{c}_{1}$-independent, we obtain, by standard forking calculus together with the fact that forking strictly lowers $D$-rank, a contradiction to each $\left(\hat{c}_{i}, b_{i}\right)$ forking with $\left(d_{1}, \ldots, d_{n}\right)$ over $\hat{c}_{1}(i=$ $2, \ldots, n+2)$. This contradiction proves the claim. It is now easy, as in the proof of Lemma 5.6, to show the type-definability of $\neg S_{\psi}$ on $\hat{q} \times q$.

We now complete the proof of Lemma 5.3.

By Lemmas 5.6, 5.7 and compactness, there is, for each $\psi \in \Psi$ a formula $\rho_{\psi}(\hat{x}, y)$ defining $S_{\psi}$ on $\hat{q} \times q$. Note that

$$
F(\hat{x}, \hat{y}) \cup q(z) \models \rho_{\psi}(\hat{x}, z) \leftrightarrow \rho_{\psi}(\hat{y}, z) .
$$

Thus there is a formula $\pi_{\psi}(z) \in q$ such that

$$
F(\hat{x}, \hat{y}) \models \forall z\left(\pi_{\psi}(z) \rightarrow\left(\rho_{\psi}(\hat{x}, z) \leftrightarrow \rho_{\psi}(\hat{y}, z)\right)\right) .
$$

Let $E_{\psi}(\hat{x}, \hat{y})$ be the definable equivalence relation given by the formula on the right hand side. So $\hat{a} / E_{\psi} \in \operatorname{dcl}(\hat{a} / F)$ for every $\psi \in \Psi$. Note that if $E_{\psi}(\hat{a}, \hat{b})$ holds for all $\psi$, then $S_{\psi}(\hat{a}, b)$ holds for all $\psi$ whereby $a / E=b / E$. Let $\tilde{a}=\left(\left(\hat{a} / E_{\psi}\right): \psi \in \Psi\right)$. So $a / E \in d \operatorname{cl}(\tilde{a})$. As we saw a couple of lines above, $\tilde{a} \in \operatorname{dcl}(\hat{a} / F)$, whereby $R(\tilde{a} /(a / E)) \leq R((\hat{a} / F) /(a / E))$ (by 4.6 (ii)), and the latter is by Fact $5.4($ iv $)<\alpha$. So by the induction hypothesis (as $\tilde{a}$ is a sequence of imaginaries and $S U(\tilde{a})<\infty$ ), $a / E$ is equivalent to a sequence of imaginaries. This completes the proof of Lemma 5.3 .

We give some consequences of Theorem 5.1.

Corollary 5.8 ( $T$ supersimple). Let $p(x)$ be an amalgamation base, where $x$ is a single imaginary variable (for example a finite tuple of real or imaginary variables). Then $C b(p)$ is equivalent to a sequence of imaginary elements (in other words $C b(p)$ exists in $\left.\bar{M}^{e q}\right)$.

Proof. By 2.24, $C b(p) \in \operatorname{dcl}\left(a_{1}, \ldots, a_{n}\right)$ for some $n$ where $\left(a_{i}\right)_{i}$ is a Morley sequence in $p$. So the result follows from Theorem 5.1 . 
Corollary 5.9 ( $T$ supersimple). Any hyperimaginary element $e$ is equivalent to a sequence of imaginaries. (Namely any type-definable over $\emptyset$, equivalence relation $E(x, y)$ on a possibly infinitary complete type $q(x)$ is equivalent on $q(x)$ to a conjunction of definable equivalence relations.)

Proof. We may assume that $t p(a /(a / E)) \models L \operatorname{stp}(a /(a / E))$ (by 3.8). Let $a=$ $\left(a_{i}: i \in I\right)$ and for each finite subset $J$ of $I$ let $a_{J}=\left(a_{i}: i \in J\right)$. Easily $\operatorname{tp}\left(a_{J} /(a / E)\right) \models L \operatorname{stp}\left(a_{J} /(a / E)\right)$ for each $J$. By Corollary 5.8, $C b\left(t p\left(a_{J} /(a / E)\right)\right)$ is a set $C_{J}$ of imaginary elements. It is easy to see that $\bigcup_{J} C_{J}=C b(t p(a /(a / E)))$.

Corollary $\mathbf{5 . 1 0}$ ( $T$ supersimple). For any amalgamation base $p, C b(p)$ exists in $\bar{M}^{e q}$.

Corollary 5.11 ( $T$ supersimple). Any complete type $p(x)$ (in possibly infinitely many real or imaginary variables) over an algebraically closed set in $\bar{M}^{\text {eq }}$ is an amalgamation base.

(Restatements: (1) The Independence Theorem holds over any algebraically closed set in $\bar{M}^{e q}$. (2) For any set $A \subset \bar{M}^{e q}$ and any tuple a of real or imaginary parameters, $\operatorname{stp}(a / A)$ implies $\operatorname{Lstp}(a / A)$.)

Proof. We prove (2). Let $e$ be a hyperimaginary in $b d d(A)$. By Corollary 5.9, $e$ is equivalent to a sequence $\left(a_{i}\right)_{i}$ of imaginaries, each of which must be in $\operatorname{acl}(A)$. Thus $a c l^{e q}(A)$ is equivalent to $b d d(A)$. So $\operatorname{stp}(a / A)$ implies $t p\left(a / a c l^{e q}(A)\right)$ implies $\operatorname{tp}(a / b d d(A))$ implies $\operatorname{Lstp}(a / A))$.

In recent work ([11]) B. Kim has used Corollary 5.10 to give a nice description of canonical bases in supersimple theories: Suppose, for example, that $p(x)$ is a canonical type (namely $C b(p)=$ domain of $p$ ). Let $\mathbf{P}$ be the family of nonforking extensions of $p$ over $\bar{M}$. Let $\Phi$ be the collection of $L$-formulas $\phi(x, y)$ such that for all $q, r \in \mathbf{P}, q|\phi=r| \phi$. Then one can show that for $\phi(x, y) \in \Phi$, this common $\phi(x, y)$ type is definable. What Kim shows is that $C b(p)$ is exactly the set of these $\phi(x, y)$-definitions, for $\phi \in \Phi$.

The nice phenomenon here is that, in this supersimple context one recovers a trace of definability of types, first going via elimination of hyperimaginaries and then existence of canonical bases as imaginaries. On the other hand in classical stability theory, the order was reversed: definability of types was the starting point, from which first existence of canonical bases, and then elimination of hyperimaginaries were deduced.

\section{REFERENCES}

1. S. Buechler, Lascar strong types in some simple theories, Journal of Symbolic Logic, 64(1999), 817-824.

2. S. Buechler, Canonical bases in some supersimple theories, preprint 1998.

3. B. Hart and A. Pillay, A note on canonical bases, preprint 1998.

4. B. Hart, B. Kim and A. Pillay, Coordinatization and canonical bases, Journal of Symbolic Logic, 65(2000), 293-309.

5. E. Hrushovski, Pseudofinite fields and related structures, preprint 1991.

6. E. Hrushovski, Simplicity and the Lascar group, preprint 1997.

7. B. Kim, Simple first order theories, Ph.D. thesis, University of Notre Dame, 1996.

8. B. Kim, Forking in simple unstable theories, Journal of London Math. Soc., 1999. MR 2000a:03052

9. B. Kim, Recent results on simple first order theories, Proceedings Blaubeuren, London Math. Soc. Lecture Notes, Cambridge Univ. Press, 1997. MR 2000e:03100 
10. B. Kim, A note on Lascar strong types in simple theories, Journal of Symbolic Logic, 63 (1998), 926-936. MR 2000a:03053

11. B. Kim, Simplicity and stability in there, to appear in Journal of Symbolic Logic.

12. B. Kim and A. Pillay, Simple theories, Annals of Pure and Applied Logic, 88 (1997). MR 99b:03049

13. D. Lascar and A. Pillay, Hyperimaginaries and automorphism groups, to appear in Journal of Symbolic Logic.

14. A. Pillay and B. Poizat, Pas d'imaginaires dans l'infini, Journal of Symbolic Logic, 52 (1987), 400-403. MR 88j:03019

15. Z. Shami, A natural finite equivalence relation definable in low theories, to appear.

16. S. Shelah, Simple unstable theories, Annals of Math. Logic, 19 (1980), 177-203. MR 82g:03055

Department of Mathematics, University of Notre Dame, Notre Dame, Indiana 465565683

E-mail address: buechler.1@nd.edu

Department of Mathematics, University of Illinois at Urbana-Champaign, 1409 W. Green Street, Urbana, Illinois 61801

E-mail address: pillay@math.uiuc.edu

Mathematical Institute, Oxford University, Oxford, England

Current address: Institut Girard Desargues (Lyon I), Université Claude Bernard, 43, Boulevard du 11 Novembre 1918, 69622 Villeurbanne-Cedex, France

E-mail address: wagner@desargues.univ-lyon1.fr 